arthralgiae 25 (39\%), tendinitis 3 (4.7\%), sacroiliitis 1 (1.6\%) and afterwards the involvement of cardiovascular system ? thrombophlebitis 11 (17\%), thrombosis v. cavae inferior 2 (3.1\%), v. cavae superior $1(1.6 \%)$, involvement of cardiac structures $4(6.2 \%)$. Central nervous system was involved in ten patients: meningoencephalitis in 2 ? $3.1 \%$, pyramidal and extrapyramidal syndrome in 1 ? $1.6 \%$, thrombosis of venous sinuses 1 ? 1.6\%. Involvement of cranial nerves was found in 3 $(4.7 \%)$ patients and peripheral neuropathy of sensorial type in 4 (6.2\%). Pulmonary vasculitis was confirmed in one patient ? 16 year old girl in the acute phase of the disease. Enterocolitis was developed in $5(7.8 \%)$ and orchiepididimytis in $2(3.1 \%)$.

In the acute phase of the disease all patients were treated with glucocorticoids and in case of multisystemic manifestations firstly with ?pulse? dose of methylprednisolone three days and afterwards cyclophosphamide in ?pulse? doses one monthly during 6 months and it was administered every 3 months until one year. A total of 19 patients were treated with cyclosporin A. Colchicin was administered in acute phase of the disease in patients with ocular involvement. Antieggregation treatment (Aspirin) was also administered.

Conclusion This paper confirms that Behcet-Adamantiades syndrome is not only three-symptom disease but also systemic generalised vasculitis. Multisystemic manifestations developed due to systemic vasculitis.

\section{AB0101 TRANSVERSE MYELITIS: A RARE MANIFESTATION OF BEHÇET'S DISEASE}

C Macieira, C Miranda Rosa, JC Teixeira da Costa, M Viana Queiróz. Unit of Rheumatology and Metabolic Bone Diseases, Santa Maria Hospital, Lisbon, Portugal

\subsection{6/annrheumdis-2001.208}

Background The diagnosis of Behçet's disease is based on wellknown criteria. Although skin, eye and joints are the most frequently involved organs, other systems may be affected such as the nervous system. Transverse myelitis is a rare and serious manifestation of the disease.

We present the case of a 51 year-old woman with a 2 year diagnosis of Behçet's disease, initially presented as oral aphtosis, genital ulcerations, symmetrical polyarthritis and a maculo-papular hand rash. Eye examination was normal.

The patient remained well for 2 years, when suddenly presented with severe headache, back pain, a rapidly progressive tetraparesia and a global sensitive deficit.

Erythrocyte sedimentation rate, C-reactive protein, total blood cell count, lisozyme, angiotensin-converting enzyme and complement factors were within the normal laboratory ranges. The serologic tests for rickettsial and Lyme?s diseases were negative. The immunological study was positive for anti-Scl70 antibody and lupus anticoagulant.

Cerebrospinal fluid analysis revealed a 48 -cell count with predominance of mononuclear forms and normal cultural and pathology examinations.

Spinal cord magnetic resonance imaging (MRI) identified a cervico-thoracic lesion suggestive of transverse myelitis. The cranial MRI was normal.

Pulsed methylprednisolone ( $1 \mathrm{~g} /$ day) was initiated, with rapid clinical improvement. After 45 days, with a maintenance dose of prednisone $(30 \mathrm{mg} /$ day) the patient was doing well referring residual sensitive deficit. With a one-year follow-up and daily prednisone $(20 \mathrm{mg})$ and azathioprine $(50 \mathrm{mg})$, the patient is assymptomatic and there are no neurological sequelae.

A literature review was performed. Central nervous system involvement occurs in less than $5 \%$ of cases of Behcet's disease and in this group $69 \%$ have clinical manifestations suggestive of transverse myelitis. High methylprednisolone doses are fundamental for favourable clinical outcome.

Objectives

Methods

Results

Conclusion

\section{FRI0149 SERUM CYTOKINES AND AUTOANTIBODIES PROFILE IN RHEUMATOID ARTHRITIS AND CONNECTIVE TISSUE DISEASES ? RELATIONSHIP TO MICROVASCULAR CAPILLAROSCOPIC ABNORMALITIES}

A Kuryliszyn-Moskal, PA Klimiuk, S Sierakowski. Department of Rheumatology and Internal Diseases, Medical University of Bialystok, Bialystok, Poland

10.1136/annrheumdis-2001.209

Background Clinical manifestations of systemic vasculitis may be postulated as a consequence of the immune response abnormalities in the course of connective tissue diseases (CTD).

Objectives The aim of this study was to elucidate the significance of different antibodies and proinflammatory cytokines in the diagnosis of vasculitis in patiens with rheumatoid arthritis (RA) and CTD.

Methods Serum of 54 patients with RA and 32 with CTD were analysed for concentrations of TNF-alpha, IL-6, antinuclear antibodies (ANA) and ds-DNA using specific enzyme immunoassay. Anti-native DNA antibodies (nDNA) and anti-neutrophil cytoplasmic autoantibodies (ANCA) were assessed by indirect immunofluorescence test.

Results Fifty out of 86 patients had systemic lesions. Pathological picture of the vascular loop in nailfold capillary microscopy was found in 84 patients. In 19 patients microvascular changes were advanced, in 35-moderate and in 30-mild. Patients with advanced changes in capillary microscopy had longer disease duration $(9,7 ; \mathrm{SD}=7,4$ years) compared to patients with mild intensity of vasculitis $(6,1 ; \mathrm{SD}=5,7$ years). Significant correlation between ANA titre and disease duration, TNF-alpha serum levels and haemoglobin concentration were observed. Presence of ANCA correlated with titer of Waaler-Rose test and erythrocyte count and of dsDNA with patient age. Serum concentration of IL-6 correlated with titer of Waaler-Rose test and CRP. RA and CTD patients with clinical signs of systemic vasculitis showed significantly higher levels of TNF-alpha, IL-6 and ANA titre as compared to those without vascular involvement. ANA and ANCA were observed significantly more frequent among patients with systemic damage compare to patients without organ injury. Also ANA were twice more frequent among patients with advanced capillaroscopic abnormalities.

Conclusion These results indicate that systemic lesions in the course of RA and CTD are accompanied by abnormalities in nailfold capillary microscopy. Our findings showed correlation between presence of ANA, TNF-alpha serum levels and clinical manifestations of vasculitis. This study point to a pathogenic role of the cytokine network and autoantibodies in systemic vasculitis. 